\title{
Intoxicación por Monóxido de Carbono: Una patología poco valorada en Urgencias
}

\footnotetext{
a Servicio de Urgencias. Hospital General de Villarrobledo. Complejo Hospitalario Universitario de Albacete (España).

Correspondencia: $\mathrm{M}^{\mathrm{a}}$ Teresa Bartolomé Navarro, C/ Paseo de Circunvalación $n^{\circ}$ 88, 2J, 02006 - Albacete, España. Correo electrónico: mt.bartolome@terra.es

Recibido el 14 de junio de 2010.

Aceptado para su publicación el 16 de agosto de 2010 .
}

\author{
$\mathrm{M}^{\mathrm{a}}$ Teresa Bartolomé Navarro ${ }^{\mathrm{a}}$, Pilar Amores Valenciano ${ }^{\mathrm{a}}$, \\ Encarnación Cuesta Vizcaíno ${ }^{a}$, Nuria Gallego Giménez ${ }^{a}$
}

\begin{abstract}
RESUMEN
La intoxicación aguda por monóxido de carbono (CO) es una urgencia médica que, de no tratarse oportunamente, puede dejar considerables secuelas neurológicas o incluso provocar la muerte del paciente. El cuadro clínico depende de la intensidad de la exposición a este gas y varía según el grado de afectación de los distintos órganos involucrados. La administración de oxígeno normobárico (NBO) es el tratamiento utilizado en la mayoría de los servicios de urgencias hasta la resolución de los síntomas y la normalización de los niveles de carboxihemoglobina $(\mathrm{COHb})$, siendo dados de alta los pacientes en la práctica común sin seguimiento posterior. Presentamos el caso de un paciente que acude a urgencias por síncope y nos cuestionamos si la utilización de oxigeno hiperbárico (HBO) frente a (NBO) disminuye las secuelas neurológicas, así como la existencia de indicaciones con evidencia científica para su uso.
\end{abstract}

Palabras Clave. Intoxicación por Monóxido de Carbono, Carboxihemoglobina, Oxigenación Hiperbárica.

\section{ABSTRACT}

Carbon Monoxide poisoning: An undervalued event in the Emergency Department Carbon monoxide (CO) poisoning is a medical emergency, which if not treated properly could leave considerable neurological sequelae or even cause the death of the patient. The signs and symptoms depend on the intensity of the exposure to the gas and vary according to the degree to which the different organs are affected. Administration of normobaric oxygen (NBO) is the treatment used in most emergency services. This gas is administered until symptoms are resolved and carboxyhemoglobin $(\mathrm{COHb})$ levels have returned to normal. The patient is then discharged with no subsequent follow-up. We present the case of a patient who came to the emergency department due to syncope. We ask ourselves whether the use of hyperbaric oxygen (HBO) compared to NBO decreases neurological sequelae, and if there is scientific evidence for its use in this indication.

Key words. Carbon Monoxide Poisoning, Carboxyhemoglobin, Hyperbaric Oxygenation.

\section{INTRODUCCIÓN}

La intoxicación aguda por monóxido de carbono (CO) es una emergencia médica común y una causa frecuente de muerte intencional o accidental, sin embargo, su reconocimiento puede ser difícil ${ }^{1}$, pudiendo pasar inadvertida debido a las características de este gas (incoloro e inodoro) y a la inespecificidad de los síntomas que produce ${ }^{2}$. Puede causar lesiones en distintos órganos: corazón, y sistema nervioso (lesiones agudas o crónicas). Éstas pueden minimizarse o prevenirse tras el tratamiento con oxigeno $\left(\mathrm{O}_{2}\right)$ al $100 \%$ o la terapia de oxigeno hiperbárico (HBO) ${ }^{1}$. 
La toxicidad de este gas es debida a la alta afinidad de la hemoglobina $(\mathrm{Hb})$ por el $\mathrm{CO}$, formando la carboxihemoglobina ( $\mathrm{COHb})$, incapaz de transpor$\operatorname{tar} \mathrm{O}_{2}$ y produciendo hipoxia tisular ${ }^{3}$. Los niveles de $\mathrm{COHb}$ dependen de múltiples factores, incluida la magnitud de la exposición, el grado de ventilación alveolar, el volumen sanguíneo y la actividad metabólica. Un nivel de $\mathrm{COHb}$ superior al $3 \%$ en los no fumadores o superior al $10 \%$ en los fumadores confirma la exposición ${ }^{4}$.

\section{OBSERVACIONES CLÍNICAS}

Paciente de 26 años remitido a Urgencias tras ser encontrado en su domicilio inconsciente. Como antecedente de interés destaca brucelosis a los 4 años. A su llegada se encontraba confuso, con tendencia a la somnolencia y con amnesia del episodio. Tan sólo recordaba haber estado toda la mañana en nuestro servicio acompañando a su mujer hasta que fue dada de alta (ésta presentaba un cuadro inespecífico de mareo y episodios al parecer no filiados estudiados con anterioridad en otro servicio de urgencias, sin llegar a un diagnóstico definitivo). No recordaba nada más.

A su llegada presentaba TA $120 / 70$, Saturación $0_{2}$ $100 \%$, FC 79 Ipm, GCS 15, PICNR, motor normal, $\mathrm{T}^{\mathrm{a}} 36^{\circ}$ y contusión en arco supraciliar derecho. El resto de la exploración física fue normal. Las pruebas complementarias mostraron creatinina $1,1 \mathrm{mg} /$ dl, GOT 33 U/L, CK 985 U/L (CK-MB 42) y Tnl $0,27 \mathrm{ng} / \mathrm{mL}$, siendo el resto de parámetros normales, incluyendo hemograma y coagulación. EI ECG no presentaba alteraciones. Se realizó TAC craneal que fue normal.

Durante su estancia en Urgencias, el paciente se mantuvo estable. Presentó un pico febril, pero la clínica neurológica fue remitiendo lentamente. En el ECG apareció inversión de la onda T de V4 a V6, menor a $1 \mathrm{~mm}$, con el paciente asintomático.

El diagnóstico diferencial era complejo: proceso infeccioso de origen cardíaco (miocarditis, pericarditis, etc.), de origen neurológico (encefalitis, meningitis, etc.), síncope de probable origen cardiogénico, cuadro comicial con periodo postictal, rabdomiolisis por decúbito prolongado, TCE leve, ingesta de tóxicos, etc. Se continúo interrogando al paciente buscando posibles causas de su cuadro clínico e informó que su caldera era muy vieja y creía no realizar una correcta combustión. Se solicitó gasometría arterial (GAB) sospechando una intoxicación por monóxido de carbono, con el resultado de $\mathrm{pH} 7,45, \mathrm{pCO}_{2} 28,8$,
$\mathrm{pO}_{2} 109,6$, Bicarbonato 19,6 y $\mathrm{COHb} 24,1 \%$, confirmándose el cuadro.

Se inició oxigenoterapia con mascarilla-reservorio, consiguiéndose normalización de cifras de $\mathrm{COHb}$. La elevación de Tnl y CK mostró picos máximos de 0,64 y 2.131 respectivamente. Su evolución fue favorable, mejorando la situación neurológica, remitiendo la clínica y normalizándose los parámetros analíticos. Fue dado de alta en los días posteriores.

\section{COMENTARIOS}

Los pacientes que han sufrido intoxicación por $\mathrm{CO}$ pueden presentar síntomas diversos e inespecíficos: problemas respiratorios, náuseas y vómitos, dolor torácico, coma, mareo, somnolencia, cefalea, arritmias cardiacas, etc. Estos síntomas suelen mejorar con la administración de oxígeno normobárico (NBO), quedando normalmente asintomáticos al alta en los servicios de Urgencias ${ }^{4}$.

Estos pacientes no suelen ser revisados, no se les realiza seguimiento post-alta y en un $20-30 \%$ de los casos, según distintas casuísticas ${ }^{5}$, presentan un Síndrome Neurológico Tardío (SNT), síndrome que aparece entre los días 2 y 28 tras la intoxicación, con frecuencia tras un periodo de normalidad. Este síndrome comprende: neuropatía periférica, alteraciones de la personalidad, de la conducta, de la memoria, de la marcha, etc. No existen datos predictivos para saber quién va a desarrollar el síndrome, siendo más frecuente en intoxicaciones graves y en ancianos ${ }^{5}$. La incidencia de las secuelas no se conoce claramente. Estudios prospectivos de pacientes tratados con NBO mostraron que el 34\% presentó síntomas como dolores de cabeza o problemas de memoria a las 4 semanas y el $46 \%$ tenía secuelas neuropsicológicas a las 6 semanas, sin embargo, entre todos los pacientes intoxicados, se desconoce el número de los que desarrollan secuelas ${ }^{4}$.

El cerebro y el corazón pueden verse gravemente afectados después de una exposición a niveles superiores al $20 \%$ de $\mathrm{COHb}$. Predominan lesiones en corteza cerebral, sustancia blanca y ganglios basa${ }^{l e{ }^{2,6}}$. Pueden exacerbar patología cardiaca incluso en personas con coronarias normales, debiendo someterse a los pacientes a un estudio cardiológi$\mathrm{CO}^{4}$.

El papel del HBO en el tratamiento de la intoxicación por CO sigue siendo controvertido. No queda claro en las distintas revisiones realizadas la mayor eficacia del tratamiento con HBO para reducir la incidencia de secuelas neurológicas en comparación 


\section{Indicaciones de terapia $\mathrm{HBO}$}

- Inconsciencia transitoria o prolongada

- Signos neurológicos

- Disfunción cardiovascular

- Acidosis grave

- Edad mayor de 36 años con exposición $\geq 24$ horas (incluyendo la exposición intermitente), o con un nivel de $\mathrm{COHb} \geq 25 \%$

- No existe una indicación clara en pacientes embarazadas, pero se debe tener en cuenta que la mortalidad fetal es superior al $50 \%$ en casos de envenenamiento severo

Tabla 1. Indicaciones de terapia HBO de la Sociedad de Medicina Hiperbárica.

con NBO (nivel de recomendación C) ) $^{7,8}$. La Sociedad de Medicina Hiperbárica recomienda la terapia con HBO en pacientes que presenten intoxicación grave y determinadas manifestaciones clínicas ${ }^{5}$ (tabla 1).

Por tanto, la realización de pruebas diagnósticas de imagen (TAC craneal), ECG y seriación de enzimas cardíacos en Urgencias es conveniente. La respuesta al tratamiento habitualmente es buena, aunque no debería olvidarse realizar el seguimiento post-alta, para detección de posibles secuelas. Los estudios publicados sobre ventajas del tratamiento con HBO muestran deficiencias metodológicas evidentes, sin poder establecerse actualmente directrices firmes sobre el uso de HBO. Sin embargo, sí se deberían tener en cuenta las indicaciones de oxigenoterapia hiperbárica descritas.

\section{BIBLIOGRAFÍA}

1 Balzan MV, Agius G, Galea Debono A. Carbon monoxide poisoning: easy to tread but difficult to recognize. Postgrad Med J. 1996; 72:470-3.
2 Prockop LD, Chichkova RI. Carbon monoxide intoxication: an updated review. J Neurol Sci. 2007; 15:122-30.

3 Croccinelli M, Fernández ME, Martins LV, Nieto MM, Yanicelli MT. Intoxicación por monóxido de carbono. Servicio de Toxicología del Hospital Pedro de Elizalde. Disponible en: www.elizalde.gov.ar/area medica/Normas/intox co.doc.

4 Lindell K, Weaver MD. Carbon Monoxide Poisoning. The New England Journal of Medicine. 2009; 19:1217-25.

5 Vila JF, Meli FJ, Serqueira OE, Pisarello J, Carrión M, Subottina N, Lylyk P. Síndrome Neurológico Tardío por envenenamiento con monóxido de carbono: Correlación clínico radiológica. Revista Neurológica Argentina. 2005; 30:118-23.

6 Ruiz-Miyares F, Deleu D, Mesraoua B, Al-Hail H, Akhtar N, D'Souza A et al. Carbon monoxide poisoning, rhabdomyolysis, cerebral petechiae and fatal course. Rev Neurol. 2008; 47:558-9.

7 Kao LW, Nañagas KA. Toxicity associated with carbon monoxide. Clin Lab Med. 2006; 26(1):99-125.

8 Wolf SJ, Lavonas EJ, Sloan EP, Jagoda AS. American College of Emergency Physicians Clinical Policies Subcommittee (Writing Committee) on Critical Issues in the Management of Adult Patients Presenting to the Emergency Department with Carbon Monoxide Poisoning. Clinical policy: critical issues in the management of adult patients presenting to the emergency department with acute carbon monoxide poisoning. J Emerg Nurs. 2008; 34:19-32. 